\title{
Tracking recurrence of correlation structure in neuronal recordings
}

\author{
Samuel A. Neymotin ${ }^{\mathrm{a}, *}$, Zoe N. Talbot ${ }^{\mathrm{d}, \mathrm{e}}$, Jeeyune Q. Jung ${ }^{\mathrm{f}}$, André A. Fenton ${ }^{\mathrm{a}, \mathrm{d}}$, \\ William W. Lytton ${ }^{\mathrm{a}, \mathrm{b}, \mathrm{c}, \mathrm{f}}$ \\ a Department of Physiology \& Pharmacology, SUNY Downstate, United States \\ ${ }^{\mathrm{b}}$ Department of Neurology, SUNY Downstate, United States \\ ${ }^{c}$ Department of Neurology, Kings County Hospital Center, United States \\ d Center for Neural Science, New York University, United States \\ e Graduate Program of Neuroscience and Physiology, NYU School of Medicine, United States \\ ${ }^{\mathrm{f}}$ Whitman Center, Marine Biological Laboratory, United States
}

\section{H I G H L I G H T S}

- PCo, a multiscale method, determines the recurrence of neural correlation structure.

- PCo operates at multiple temporal and spatial scales without dimensional reduction.

- PCo detects different place cell ensemble states which represent the environment.

- PCo reveals anomalous brain states in field potentials from an animal epilepsy model.

\section{A R T I C L E I N F O}

\section{Article history:}

Received 26 August 2016

Received in revised form 7 October 2016

Accepted 11 October 2016

Available online 13 October 2016

\section{Keywords:}

Correlation structure

Temporal recurrence

Multiscale analysis

Neuronal ensembles

Local field potentials

\begin{abstract}
A B S T R A C T
Background: Correlated neuronal activity in the brain is hypothesized to contribute to information representation, and is important for gauging brain dynamics in health and disease. Due to high dimensional neural datasets, it is difficult to study temporal variations in correlation structure.

New method: We developed a multiscale method, Population Coordination (PCo), to assess neural population structure in multiunit single neuron ensemble and multi-site local field potential (LFP) recordings. PCo utilizes population correlation (PCorr) vectors, consisting of pair-wise correlations between neural elements. The PCo matrix contains the correlations between all PCorr vectors occurring at different times. Results: We used PCo to interpret dynamics of two electrophysiological datasets: multisite LFP and single unit ensemble. In the LFP dataset from an animal model of medial temporal lobe epilepsy, PCo isolated anomalous brain states, where particular brain regions broke off from the rest of the brain's activity. In a dataset of rat hippocampal single-unit recordings, PCo enabled visualizing neuronal ensemble correlation structure changes associated with changes of animal environment (place-cell remapping).

Comparison with existing method(s): PCo allows directly visualizing high dimensional data. Dimensional reduction techniques could also be used to produce dynamical snippets that could be examined for recurrence. PCo allows intuitive, visual assessment of temporal recurrence in correlation structure directly in the high dimensionality dataset, allowing for immediate assessment of relevant dynamics at a single site. Conclusions: PCo can be used to investigate how neural correlation structure occurring at multiple temporal and spatial scales reflect underlying dynamical recurrence without intermediate reduction of dimensionality.
\end{abstract}

(c) 2016 The Author(s). Published by Elsevier B.V. This is an open access article under the CC BY-NC-ND license (http://creativecommons.org/licenses/by-nc-nd/4.0/).

\section{Introduction}

Brain states are hypothesized to provide snapshots of ongoing neural and functional dynamics across multiple temporal and

\footnotetext{
* Corresponding author at: 450 Clarkson Ave, Box 31, Bklyn, NY 11203-2098, United States.

E-mail address: samn@neurosim.downstate.edu (S.A. Neymotin).
}

spatial scales. Analysis of large-scale brain dynamics has revealed that such patterns occur during a resting state (default mode), forming a complex network across regions (Greicius et al., 2003, 2009; Hebb, 2002; von der Malsburg, 1981; Allen et al., 2012; Chen et al., 2013; Lu et al., 2007). Considerable research has been performed at the macroscale using fMRI to determine these recurring connectivity patterns and their relation to cognitive function (Gilbert and Sigman, 2007; Hagmann et al., 2008; Lungarella and Sporns, 2006; Sporns and Zwi, 2004) and to disease (de Haan 
et al., 2012; Kühn and Gallinat, 2013; Moncrieff and Cohen, 2006; Uddin et al., 2014). Oscillatory states provide one set of operational modes which occur in thalamocortical and hippocampal systems at multiple spatial (microcircuit to brain area) and temporal scales (millisecond to minutes) (Buzsáki, 2010; Palva and Palva, 2011). Understanding the signatures and origins of these modes will form a bridge from micro- to macro-scale brain states (Colgin et al., 2009; Schomburg et al., 2014; Fukushima et al., 2012; Hunter et al., 2006; Kumar et al., 2013; Schroeder and Lakatos, 2012). Evaluating the expression of the different brain states will also enable a deeper understanding of the strategies the brain uses to perform cognitive computations - the brain's coding languages (Treisman, 1996; von der Malsburg, 1995). New techniques for high dimensional neural system evaluation will become increasingly important as new micro and macro imaging methods are developed under the auspices of the U.S. BRAIN (Brain Research through Advancing Innovative Neurotechnologies) project and related projects elsewhere in the world (Bargmann et al., 2014).

In addition to the macro level, analysis of neural systems is also done at the level of neuronal ensemble through spike-train analysis, and at the meso level through analysis of local field potentials (LFPs). At the lower levels, most previous work on neuronal spiking activity has used measures of similarity of spike patterns of single neurons in different contexts (Fellous et al., 2004). Other work includes determining the correlation of smoothed ensemble population firing vectors during distinct behavioral states such as sleep, run and rest, at behaviorally relevant time-scales. However, these methods do not take into account the single cell or local area correlation structure across ensembles (Gothard et al., 1996; Kelemen and Fenton, 2013; Louie and Wilson, 2001). Other work that does include assessment of ensemble correlation structure and dynamics in different behavioral states (Song et al., 2009, 2015; Chan et al., 2011), does not include visualization techniques that help interpret how the correlation structure recurs over time (Kudrimoti et al., 1999).

We propose a new method which enables tracking how the correlation structure of neuronal ensembles changes over time. Our method, Population Coordination, PCo, facilitates visualizing the recurrence of correlation structure of neuronal ensembles. $P C o$ is intrinsically a multiscale measure in the temporal domain since it looks at recurrence at a high time scale of a spatiotemporal pattern measured at one or more shorter time scales. PCo is tunable to allow choice of view at faster or slower time scales. It is also applicable to data recorded at different spatial scales, from ensembles of single cells or multi-site LFP recordings, as shown here, on up to larger scale recordings using EEG or other measures. Measures from different scales could also be combined. In this study, we develop the PCo method and show how it may be used to determine correlation structure in different experimental conditions. We use PCo on two databases of electrophysiological data: hippocampal place cell data and multi-site LFP activity. We demonstrate the usefulness of $P C O$ in identifying and following brain states.

\section{Materials and methods}

\subsection{Place cell electrophysiology}

Adult male LongEvans hooded rats were obtained from a commercial breeder (Taconic Farms). The rats were 350-400 g at the start of training. Two recording environments were used (Fenton et al., 2008). One was a standard apparatus for place cell recordings. It was a $68-\mathrm{cm}$-diameter gray cylinder with a polarizing card on the wall that occupied $90^{\circ}$ of arc. The other apparatus was a $150 \mathrm{~cm} \times 140 \mathrm{~cm}$ chamber with stairs on three sides. The rat could traverse the stairs to access two drinking spouts. One spout provided plain water and the other contained a sucrose solution. Access to fluid motivated the rat to traverse the stairs and to continuously forage for scattered food for $1 \mathrm{~h}$. The rat could look through the chamber bars and see into the surrounding room. The cylinder was positioned within the chamber for cylinder recordings and it was removed for chamber recordings.

Tetrodes made by twisting 4 strands of $25 \mu \mathrm{m}$ nichrome, were used for action potential recordings, which can aid unitary waveform discrimination (Gray et al., 1995; O’Keefe and Recce, 1993). Eight tetrodes were loaded into a custom machined microdrive assembly. The assembly was surgically implanted under pentobarbital anesthesia $(50 \mathrm{mg} / \mathrm{kg}$ ) to position the tetrodes above the pyramidal cell layer in the dorsal hippocampus (centered at AP: $3.8 \mathrm{~mm}$, L: $2.5 \mathrm{~mm}$, DV: $2 \mathrm{~mm}$ relative to bregma) (Paxinos and Watson, 2007). The rats were allowed to recover for at least one week before the tetrodes were individually advanced in $<50 \mu \mathrm{m}$ steps during the course of 1-2 weeks. The goal was to position as many of the eight tetrodes in the dorsal CA1 pyramidal layer at the same time.

Extracellular potentials were buffered by unitary gain amplifiers plugged into the microdrive connector on the rat's head. The buffered signals were transmitted to main amplifiers (A-M Systems, WA) along wires. Action potential $(300-10,000 \mathrm{~Hz})$ band-pass filtered signals were digitized $(32 \mathrm{kHz})$ time-stamped $(100 \mu \mathrm{s}$ resolution) and $2 \mathrm{~ms}$ tetrode waveforms were recorded using custom software (AcX, A.A. Fenton). Whenever an action potential voltage exceeded a threshold voltage the voltages on all 4 wires of the tetrode were collected. The recorded action potential waveforms were aligned with the voltage at the threshold crossing set to the sample at $250 \mu \mathrm{s}$. This alignment produced a time-axis origin for measuring time-dependent waveform features, such as the waveform voltage at a particular time point relative to the threshold crossing at $t=250 \mu \mathrm{s}$. Further details of methods were described previously (Fenton et al., 2008).

Single-unit waveform isolation was done manually using custom software (WClust A.A. Fenton, S.A. Neymotin) (Neymotin et al., 2011) that allows the user to define unitary waveform parameter clusters by drawing convex polygon boundaries in 2-dimensional (2-D) slices of waveform parameter space. The waveform parameters included the positive and negative peak amplitudes on each tetrode wire, determined from a cubic spline (Press et al., 2007) of the digitized waveform; the principal components computed either from the waveform on each tetrode wire separately, or computed from the concatenation of the four tetrode waveforms; the waveform energy between a user-selected pair of time points; the waveform voltage or slope at a user-selected time point; the waveform voltage on each tetrode wire at the time of the peak voltage on the largest waveform in the tetrode event.

\subsection{Epilepsy model}

Timed-pregnant Long-Evans rats were obtained at 13-14 days of gestation from Charles River Laboratories (Wilmington, MA). The pups received a ventral hippocampal lesion according to the procedure of Lipska et al. (1993). On postnatal day 7 (P7), pups weighing $14-18 \mathrm{~g}$ were anaesthetized by hypothermia (placed on ice for $15-20 \mathrm{~min}$ ). A puncture hole was made bilaterally on the skull for injection sites (relative to bregma AP: $-3.0 \mathrm{~mm}$, ML: $+3.5 \mathrm{~mm}$ ), and $0.3 \mu \mathrm{l}$ of ibotenic acid solution (IBO, Sigma, $10 \mu \mathrm{g} / \mu \mathrm{l}$ ) or saline (SAL, control rats) was infused bilaterally into the ventral hippocampal formation (relative to the skull surface DV: $-5.0 \mathrm{~mm}$ ) at $0.15 \mu \mathrm{l} / \mathrm{min}$. The pups were warmed and then returned to their mothers. On P21, animals were weaned. 


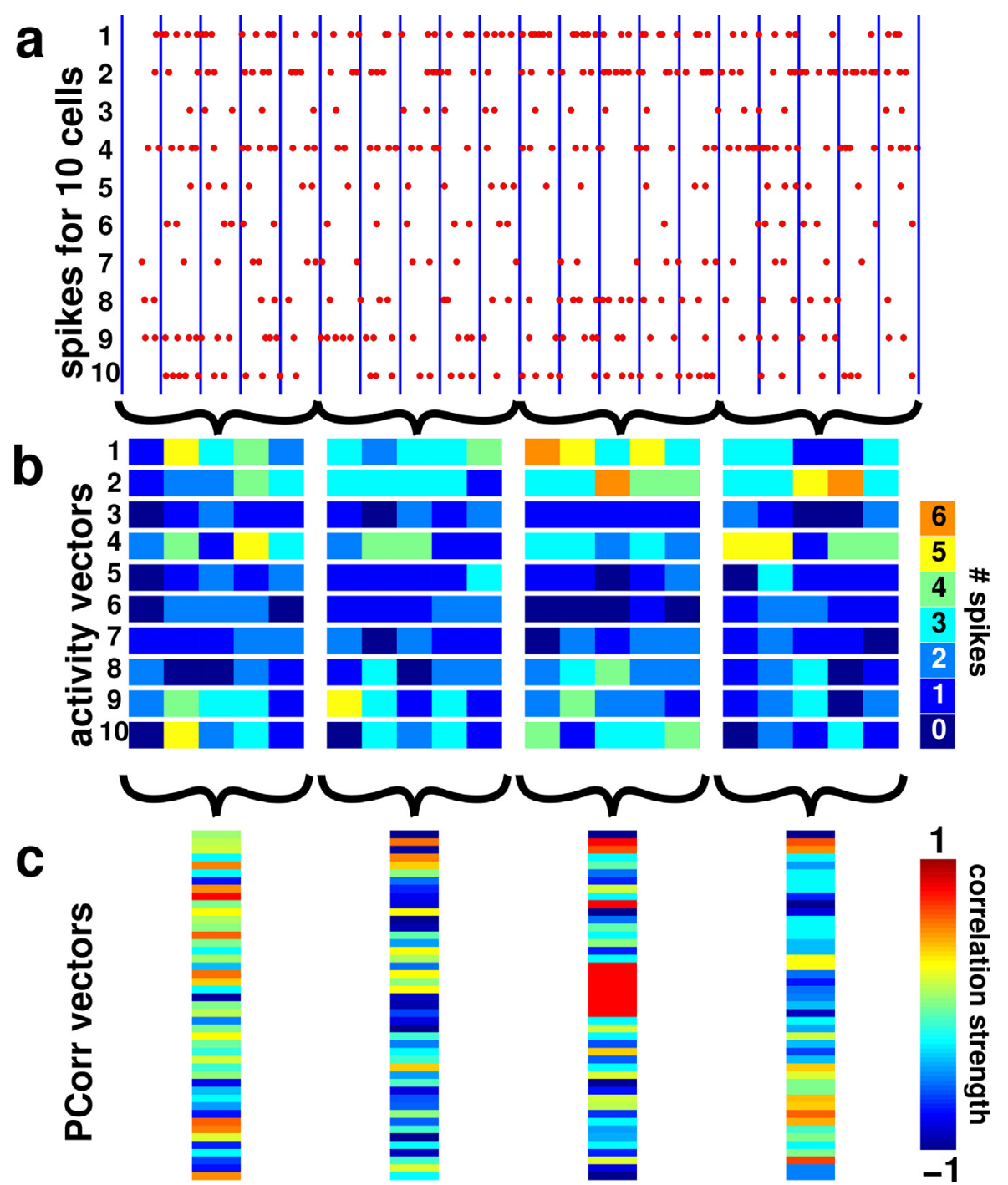

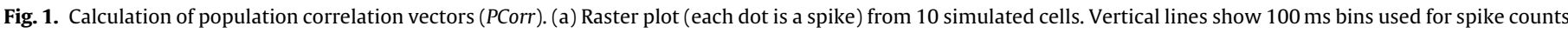

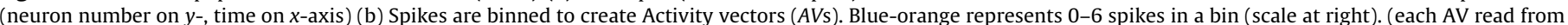

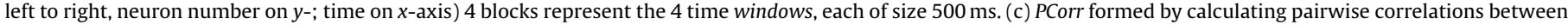

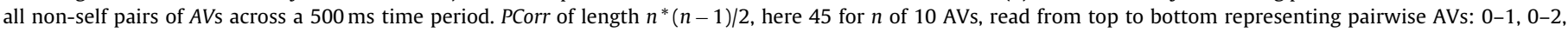
$0-3, \ldots, 7-7,7-8,7-9,8-9$. (For interpretation of the references to color in this figure legend, the reader is referred to the web version of the article.)

\subsection{EEG and local field potential (LFP) recording}

Between P60 and P90, rats were implanted with electrodes for recording local field potentials and the epidural cortical EEG as described (Lee et al., 2012; Neymotin et al., 2010). Electrodes were made by attaching $75 \mu \mathrm{m}$ Nichrome wire to contacts in Millmax connectors for LFP recordings, and a stainless-steel jeweler's screw on the rat's skull was attached to a Millmax connector by a $75 \mu \mathrm{m}$ Nichrome wire for epidural cortical EEG recordings. Recordings were made bilaterally from the following locations: piriform cortex: AP: -0.9 , ML: +5.5 , DV: 8.8 ; dorsal hippocampus: AP: -4.0 , ML: +2.5, DV: 3.0; frontal screw: AP: 0, ML: +3.0; back screw: AP: -6.0 , ML: +3.0 (all in $\mathrm{mm}$ ). All electrodes were referred to a reference electrode implanted in the cerebellar white matter. Rats were recorded in their homecages for $1 \mathrm{~h}$ at a time for a total of $12-15 \mathrm{~h}$.

8-Channel recordings of electrophysiological signals were performed. The signals were digitized (24-bits, $12 \mathrm{kHz}$ ) and transmitted wirelessly (Bio-Signal Group Corp., Brooklyn, NY). The digital signals were received by a set of dedicated digital signal processors, bandpass filtered $(1-500 \mathrm{~Hz})$, amplified digitally, and then down-sampled $(2000 \mathrm{~Hz})$.
Both studies received IACUC approval and were in accordance with institutional and NIH guidelines.

\subsection{Kendall's tau}

Kendall correlation was used for our single-unit data because other covariance measures cannot handle the extremely sparse activity vectors produced by cells that fire infrequently, giving vectors which are mostly zeros with a few scattered ones (Press et al., 2007). Kendall's $\tau$ non-parametric rank correlation (Kendall, 1938) is defined as:

$$
\tau=\frac{n_{c}-n_{d}}{(1 / 2) \cdot n \cdot(n-1)}
$$

based on concordant $\left(n_{c}\right)$ and discordant $\left(n_{d}\right)$ paired pairs. All pairs of elements in the first time-series are compared with all ordered pairs of the second time series. The two numbers in each pair can either be increasing, decreasing, or equal. Concordance or discordance of these orderings is then evaluated for the same location between the two vectors. Since firing tends to be sparse, producing many $(0,0)$ pairs, this produces many ties between the two 
time-series, which do not indicate true correlation. The many ties from a $(0,0)$ compared to another $(0,0)$ are taken into account by the normalizing denominator $(1 / 2 \cdot n \cdot(n-1))$, which represents the total number of ordered pairs in the time-series. To reduce the runtime of the calculation from $O\left(n^{2}\right)$ to $O(n \cdot \log (n))$, we used a fast version of Kendall's $\tau$ based on sorting procedures (Knight, 1966).

\section{Results}

\subsection{Recurrence of correlation structure}

Population Coordination (PCo) quantifies repetitive Population Correlation (PCorr) activity structure at multiple locations, allowing for intuitive display of temporal recurrence of specific correlation structure patterns.

We start by describing the formation of the PCorr vectors, itself a multistep process (Fig. 1). Brain Activity vectors (AVs) will either reflect local area activity (from local field potentials - LFPs), or can be formed by binning individual single-unit recordings. In Fig. 1a, spike counts in consecutive bins define the AVs in Fig. 1b, providing a snapshot of amplitude modulation in each cell. In this case we utilize two time scales: 1 . the bin size to calculate spike counts - here $100 \mathrm{~ms} ; 2$. the window size - here 5 bins $\times 100 \mathrm{~ms} /$ bin $=$ $500 \mathrm{~ms}$ - that determines the duration of amplitude modulation to be further assessed (in the case of LFPs only window size is relevant). Bin size determines "instantaneous" activity and must be long enough so as to see changes in spike counts between the successive bins, but not so long as to average out all of the modulation. In the case of LFP, dipole summation and temporal low-pass filtering effectively "bins" multi-unit activity. Window size determines the duration over which we will be looking for comodulation across signals. Conceptually, co-modulation will reflect the co-involvement of cells or brain areas (for LFPs) with particular phase relations in a particular frequency band, Hypothetically such phase relations define the grouping of cells or areas as a dynamical ensemble. These dynamical ensembles are best recognized in the case of gamma (Gray et al., 1989), but there is also evidence of tying cells of particular cortical layers together with alpha (Haegens et al., 2015; Lakatos et al., 2016).

Each PCorr relates modulation of each location (cell or LFP) to modulation at each other location during the time window. Fig. 1 shows 4 time windows, with one PCorr vector created for each 500 ms window (Fig. 1c). Each PCorr shows all amplitude modulation correlations - taking all (non-self) pairwise correlations between $n \cdot(n-1) / 2$ pairs of activity vectors. Here, 10 AVs in a time window produce a PCorr of length $10 \cdot 9 / 2=45$. Pcorr may be displayed, as here, as a 1-D vector, or may be displayed as a full correlation matrix with redundancy between upper and lower values across the diagonal. Large positive correlations are drawn in orange or red and large negative correlations are drawn in blue (color scale at right of Fig. 1c).

Population Coordination $(\mathrm{PCO})$ measures how correlation structure of neuronal ensembles recurs over time (Fig. 2). The method calculates the correlation coefficient between all pairs of PCorr vectors from different times (Fig. 2). Note that this is a second correlation step - we are now looking for correlations across correlation vectors that represent correlations of activity patterns across a time duration of window size. Here it is most natural to view the $m \cdot(m-1) / 2$ correlations of $m$ PCorr vectors as a full correlation matrix, noting again that lower and upper values across the diagonals are redundant, as illustrated in Fig. 2, bottom. In the PCo matrix, the $x, y$ axes are times so that matrix entries provide correlations between PCorr vectors at two times. The diagonal of the PCo matrix is trivially equal to 1 (dark red). Movement away from the diagonal represents increasing temporal differences between the PCorr

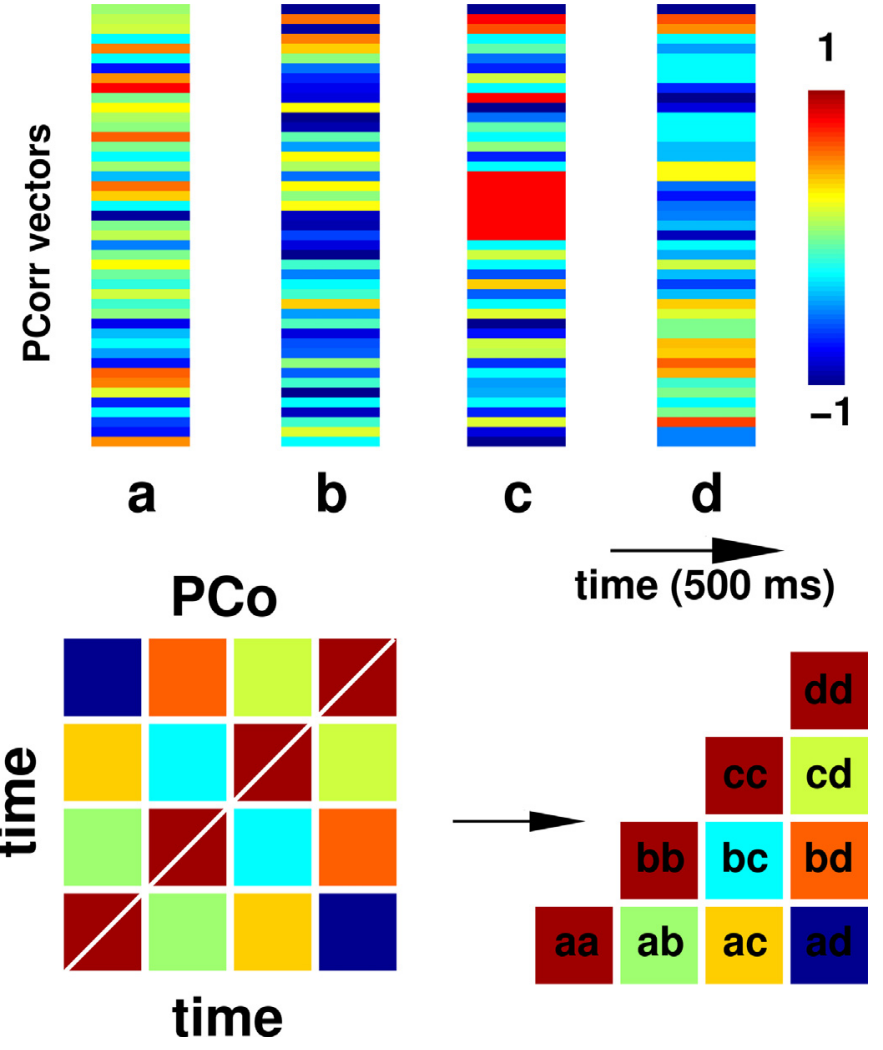

Fig. 2. Visualization of Population Coordination $(P C O)$, demonstrating recurrence of population correlation (PCorr) vectors over time. a-d are the same PCorr vectors shown in Fig. 1, which are each formed from $500 \mathrm{~ms}$ of ensemble activity. The PCo matrix shown here is formed by calculating correlations between all pairs of these PCorr vectors. $x, y$ axes both represent time, and the color values indicate correlation between the PCorr vectors at the specified times. (For interpretation of the references to color in this figure legend, the reader is referred to the web version of the article.)

vectors used in the calculation. Recurrence of similar correlation structure appears as hot spots in the matrix - e.g., the orange block marked $b d$ in Fig. 2 (lower right) shows the similarity of the PCorrs at time $b$ and time $d$, demonstrating a recurrence.

\subsection{Applications: single unit recordings and local field potentials}

The PCo method is generalizable to recordings at any spatial and temporal scales where there is interest in looking at recurring patterns of activity across a set of locations in the brain (or elsewhere). The increasing ability to record from a large number of locations provided by new innovative neurotechnologies will permit further extensions not currently possible (Bargmann et al., 2014). For example, it would be of great interest to record from multiple synapses onto a single pyramidal neuron in order to look for recurring patterns of input. The spatial scale is of course a function of the recording, currently from single unit to local field potential and electroencephalogram (EEG). We give two examples here punctate single unit spike times and continuous LFP. In the present example, we handle units by windowing, as shown in Fig. 1. However, units could be handled in various alternative manners. For example, spike phase relative to a local alpha or gamma wave could be used as the activity measure.

\subsection{Single unit analysis}

Because each place cell has a characteristic discharge correlate, called the cell's place field (O'Keefe and Dostrovsky, 1971; O'Keefe, 1979), we begin by examining the patterns of discharge amongst an 

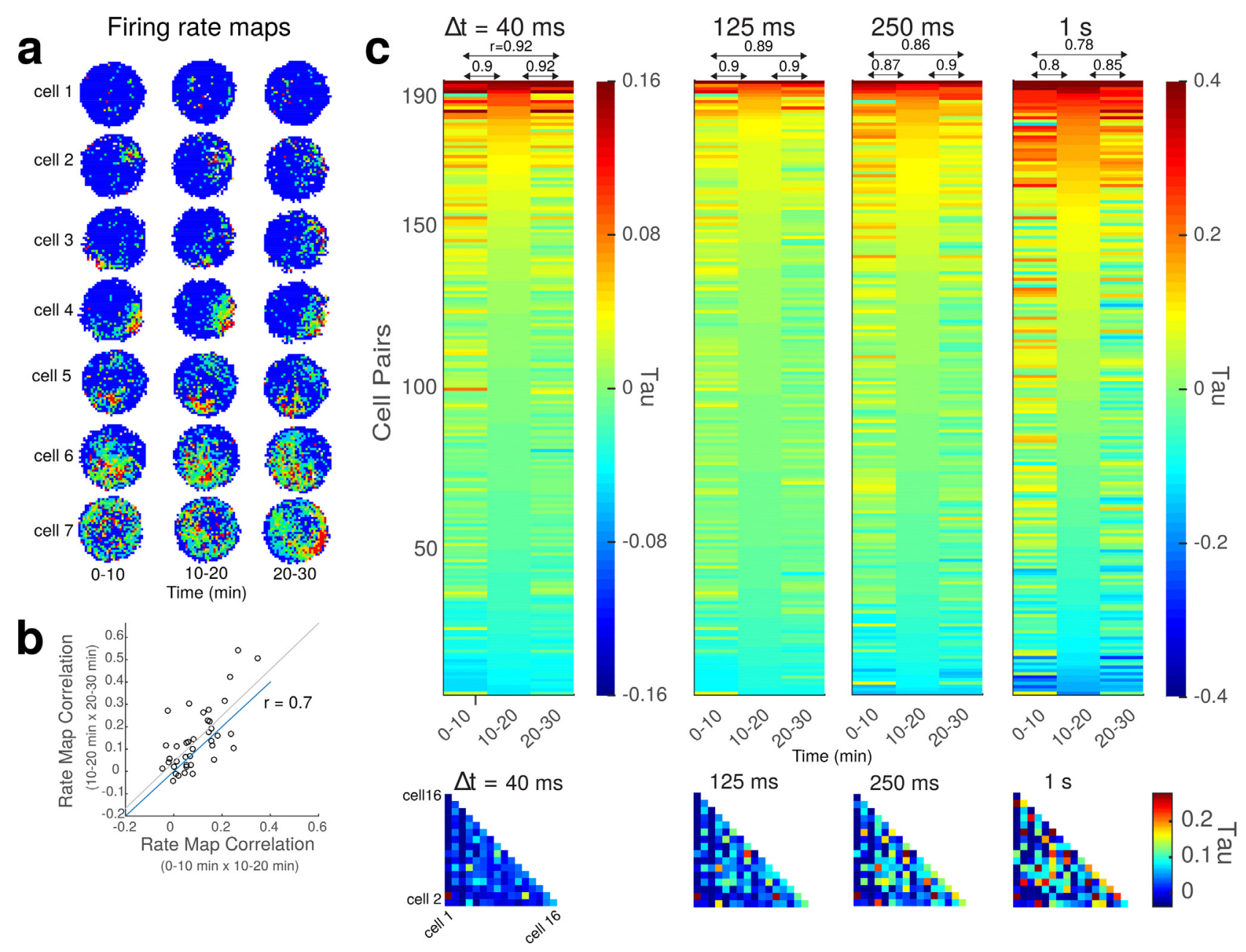

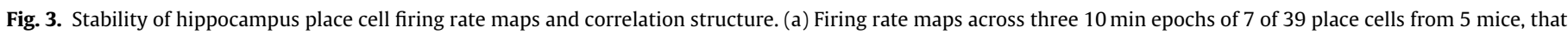

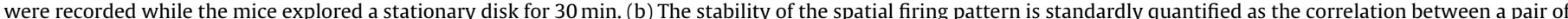

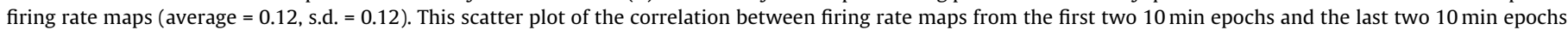

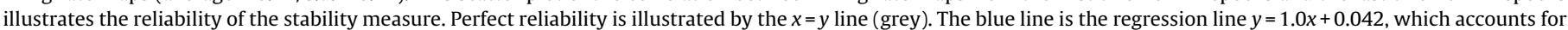

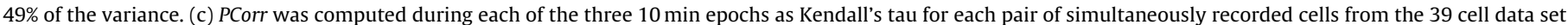

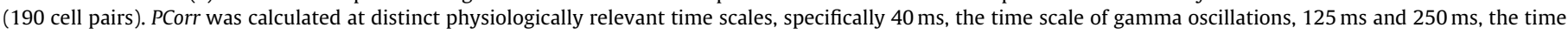

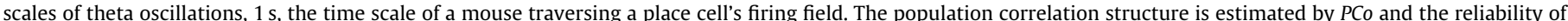

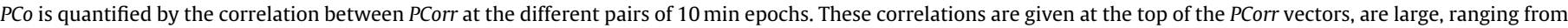

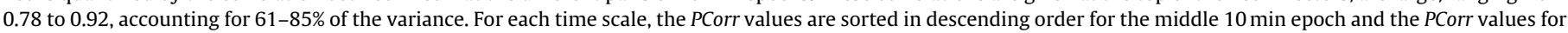

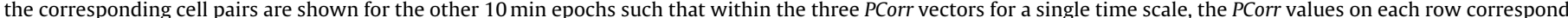

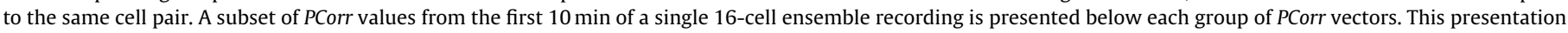

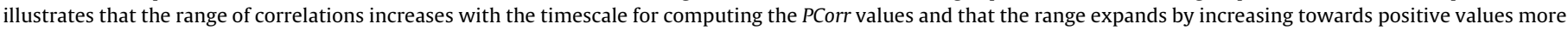
than it expands towards negative values.

ensemble of place cells. Single-unit activity was recorded from dorsal CA1 of the hippocampus in C57BL/6J mice while they explored a $40 \mathrm{~cm}$ diameter cylinder with a transparent wall. Time-averaged firing rate maps were computed for each of three $10 \mathrm{~min}$ epochs of the $30 \mathrm{~min}$ recording session (Fig. $3 \mathrm{a}$ ). The seven example cells shown are presented in decreasing order of the spatial sparseness. By inspection, the order is approximately preserved across each of the three epochs. We then quantified the stability of this representation of place cell firing. The stability across two recording epochs, quantified as the pixel-by-pixel correlation of the superimposed maps is on average $0.12+0.12$ for the data set of 39 place cells (Bostock et al., 1991; Kentros et al., 2004). The reliability of this stability estimate was evaluated by computing Pearson's correlation between the stability estimates across the first two recording epochs and the second two epochs ( $r=0.7$; Fig. $3 b$ ), accounting for approximately half of the spatial firing variance.

We then computed the set of PCorr values for the 190 simultaneously recorded cell pairs in this data set. PCorr was computed separately for each 10 min epoch and at different time scales corresponding to the main physiological mechanisms of neural coordination that have been identified to govern place cell discharge. Specifically, the $40 \mathrm{~ms}$ timescale estimated the organization of firing on the time scale of gamma oscillations whereas the $125 \mathrm{~ms}$ and $250 \mathrm{~ms}$ intervals estimated the organization by theta oscillations (Buzsáki, 2010), and $1 \mathrm{~s}$ corresponds to the time it takes for the mouse to walk across a firing field (Fenton and Muller, 1998). The PCorr values of the 190 different cell pairs are sorted in descending order according to value in the middle $10 \mathrm{~min}$ epoch, and the cell pair order is preserved across the three epochs (Fig. 3c). By inspection, the basic ordering is preserved, like in the firing rate map examples. The PCorr values range from positive to negative values, with a slight bias for positive values and the values tend to be greater for the longer time scale estimates because greater variance in firing is possible for longer time intervals. We then quantified the stability of this representation of place cell firing. The stability across each pair of recording epochs was quantified by $P C O$. This 

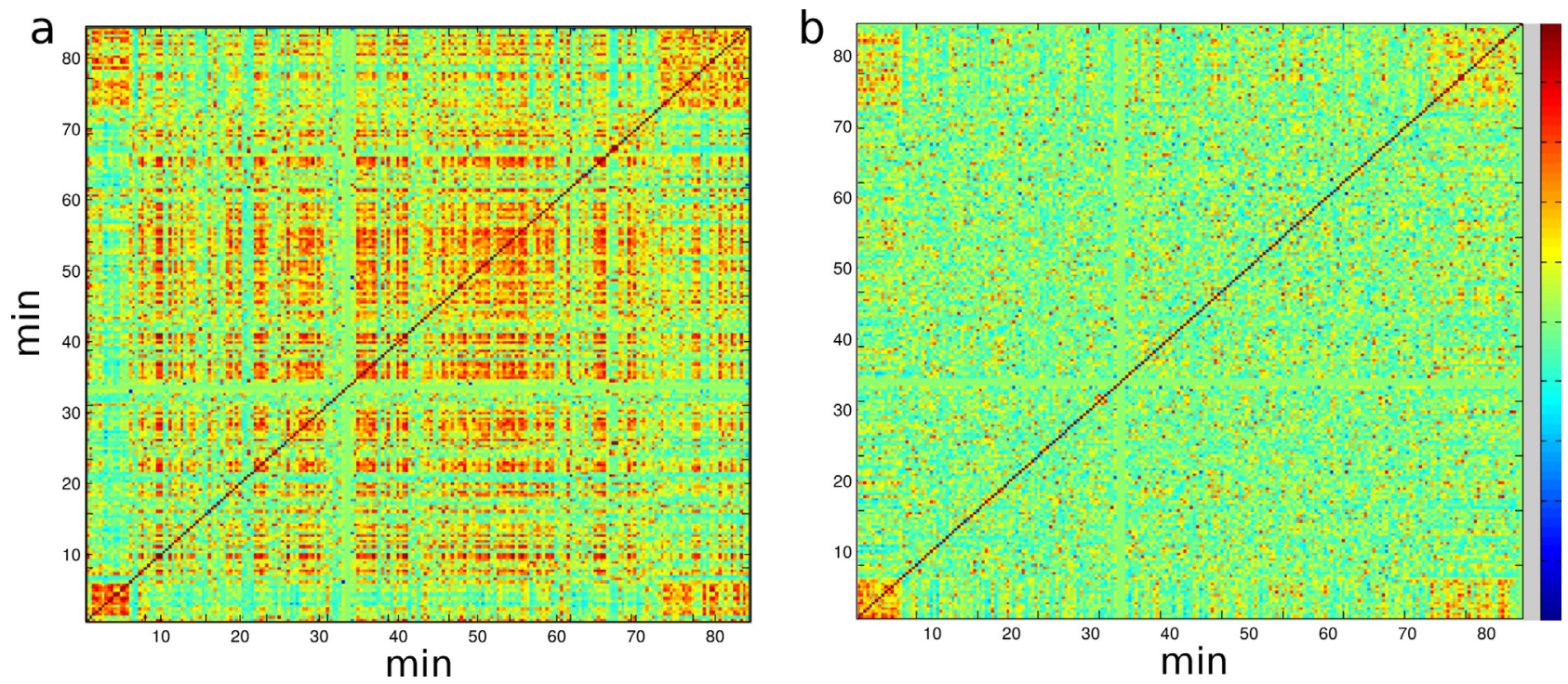

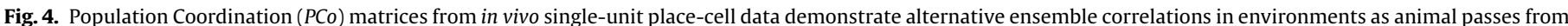

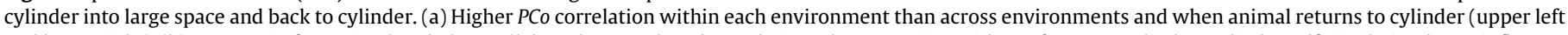

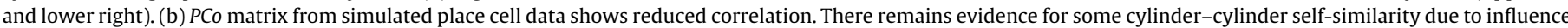

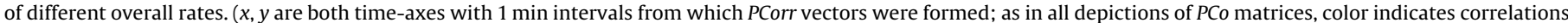

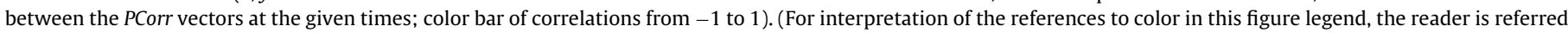
to the web version of the article.)

cell-pair-to-cell-pair correlation of the superimposed PCorr vectors was high, ranging from 0.78 to 0.92 across the different time scales. Thus $P C o$ accounts for at least as much, if not more variance (61-85\%) than the standard firing rate map representations of place cell activity.

In the next set of single-unit analyses we used in vivo hippocampal ensemble data from a prior study to further demonstrate the utility of the PCorr/PCo methods (Fenton et al., 2008). In that study, place cells were recorded in two different environments, leading to a phenomenon known as place-cell remapping when the animal is moved from one environment to the other. Remapping has typically been detected at the single cell scale by firing rate changes or place field shifting (Bostock et al., 1991; Muller and Kubie, 1987; Kelemen and Fenton, 2010, 2013). PCo permits us to look at alterations in cell coordination across an ensemble of cells recorded simultaneously. The animal was placed in either a small cylinder (CYL) or a big chamber (BC) - 6 min in the cylinder, then $65 \mathrm{~min}$ in the chamber, followed by a final $11 \mathrm{~min}$ in the cylinder. Overall these cells have low overall firing rates (typically $1-4 \mathrm{AP} / \mathrm{s}$ ) because the animal is often outside the preferred place for a given cell. For this reason we used long activity vectors: length 600 with bin size of $100 \mathrm{~ms}$, corresponding to $1 \mathrm{~min}$ of time, usually giving $0-4$ spikes in most bins. The sparse vectors then required the use of Kendall's correlation in the $P C o$ analysis.

Times in the different environments can be directly read off of Fig. 4 by noting the blocks of red that show frequent periods of self-similarity during the chamber period (central block) as well as self-similarity during the first (lower-left) and last (upper-right) cylinder periods. Additionally there is strong similarity in activity across the two periods spent in the cylinder (upper-left, lowerright). The differences in patterns across environments was due to different pairs of cells having high correlation in the two environments. PCo correlation is low between the cylinder (CYL) periods and the period spent in the $\mathrm{BC}$ - note the green horizontal stripe from 7 to $72 \mathrm{~min}$ at the bottom. Interestingly there are many stripes of non-correlation during the $\mathrm{BC}$ period, suggesting that the activity patterns are not consistently repeating during the entirety of the time in the big chamber.
Place cell firing is of course primarily associated with place high firing when the animal passes through a particular location with little firing elsewhere. This means that much of the correlation structure will simply be due to animal location, rather than to the fine structure of firing. We therefore used a Poisson point process as a control, creating inhomogeneous Poisson firing that preserved instantaneous firing rates, read from average rates at individual locations (Fig. 4b). This had the effect of giving randomized firing times (effectively shuffled) while preserving classical place cell responses. Therefore, remaining correlation between simulated place cells was simply due to overlapping firing fields, and not an explicit function of ensemble coordination. The simulated data showed lower correlation values, particularly in the $\mathrm{BC}$ condition. Correlations were still high during the CYL periods, as well as during the self comparison in periods CYL1 and CYL2. This increased activity reflected the higher spatial density in the smaller space for those place cells that were active in that space.

\subsubsection{Local field potential}

$P C o$ was used to evaluate recurrence of correlation structure in multi-channel local field potential (LFP) recordings, using $60 \mathrm{~min}$ of $2 \mathrm{kHz}$ sampled LFP from an animal model of medial temporal lobe epilepsy (Fig. 5). Piriform cortex, dorsal hippocampus, front cortical screw, and back cortical screw were recorded bilaterally, for a total of 8 channels, creating 28-dimensional PCorr vectors. Here, activity vectors were not needed since raw LFP at an individual site could be used. PCorr vectors were formed by taking the Pearson correlation between all LFP pairs, and the PCo matrix as the set of pairwise correlations among PCorr vectors.

There was considerable recurrence of PCorr vectors, with bands of high and low self-similarity seen as patches in the PCo matrix (Fig. 5a). Overall $P C o$ values were high, with an average correlation of $\sim 0.4$ (Fig. 5a; bottom). Fig. 5b shows the LFP activity and associated PCorr at two highly correlated times, indicated by blue asterisks in Fig. 5a. Note that the length-28 vector is shown here redundantly as a full correlation matrix - the elements below the diagonal are 28 unique comparisons. Most of the channels 

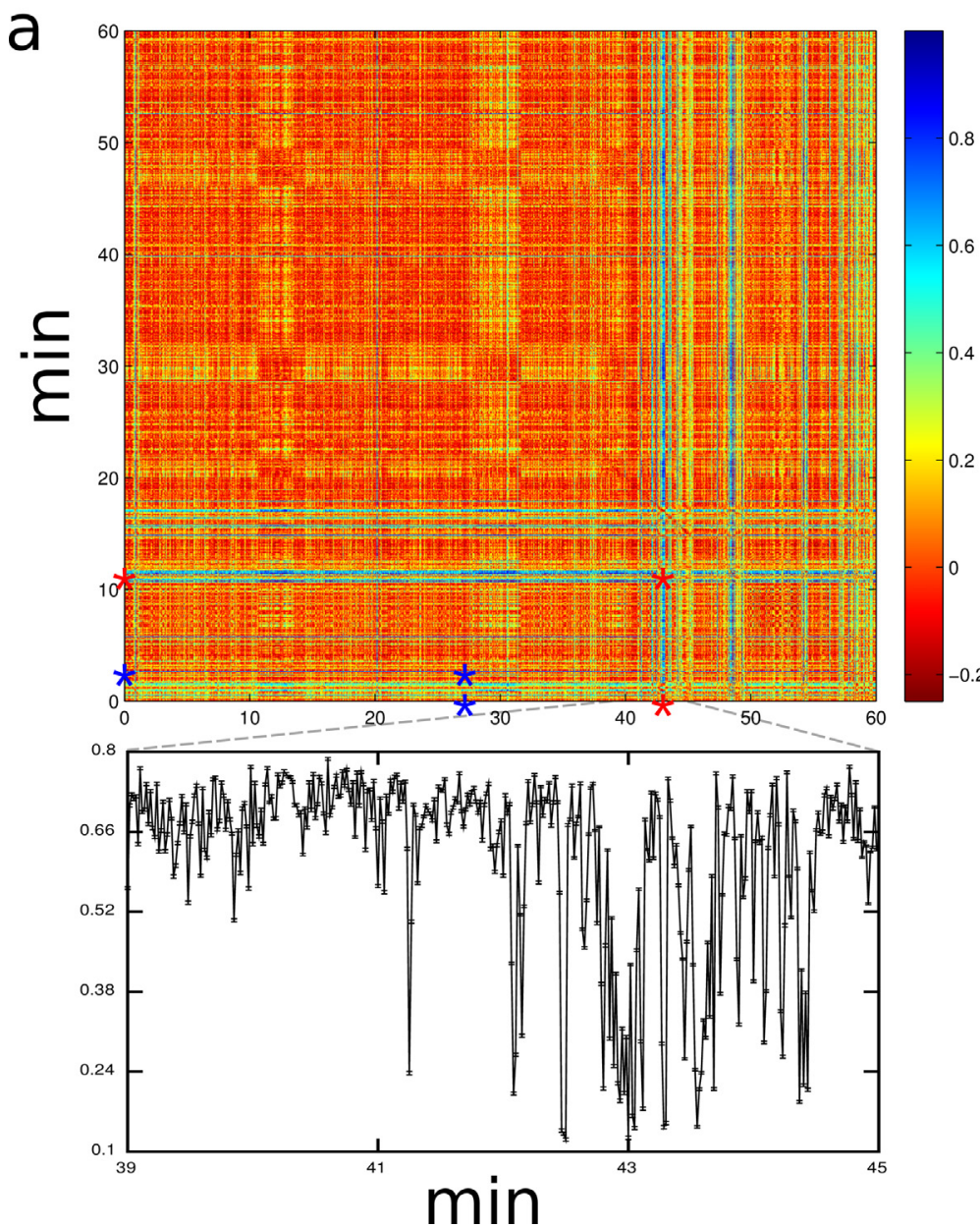

b
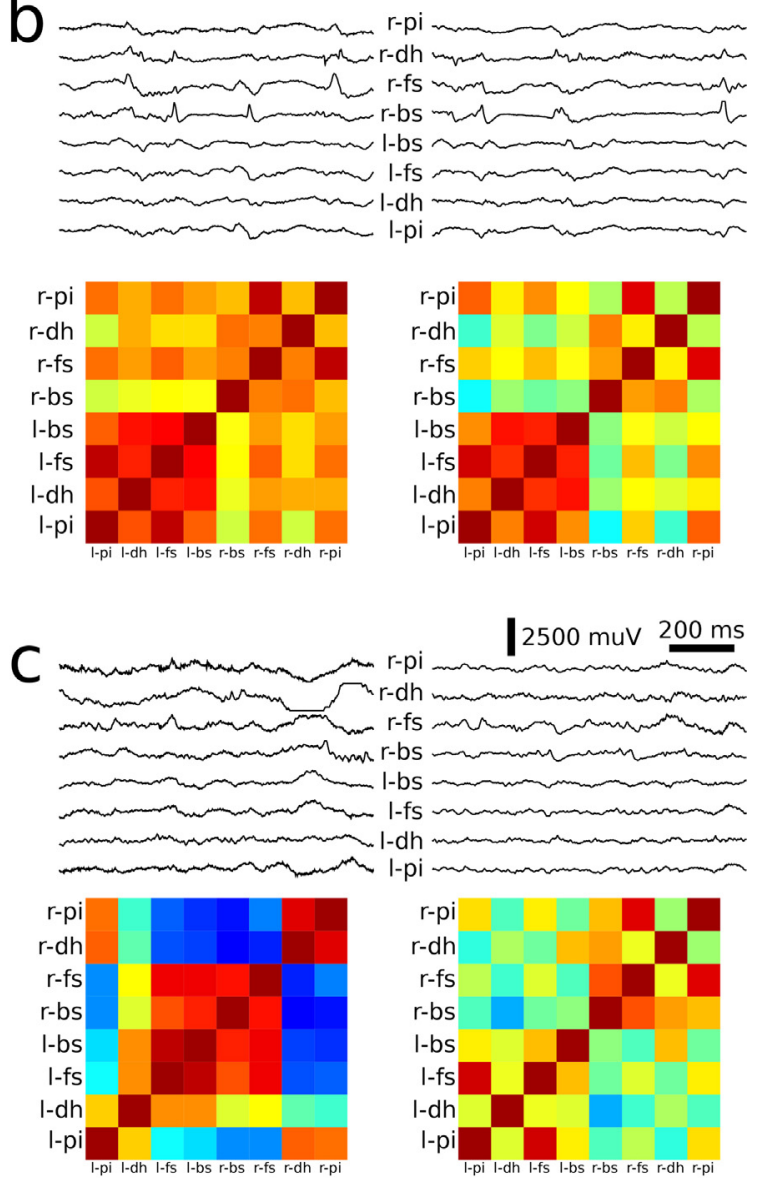

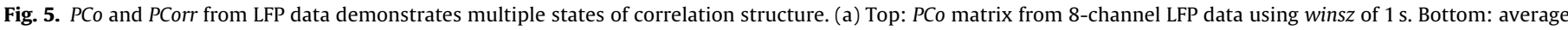

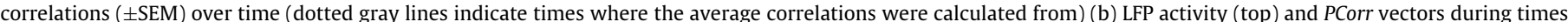

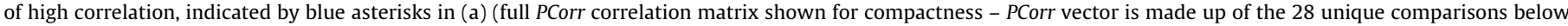

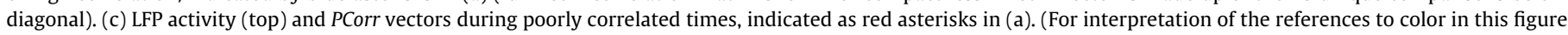
legend, the reader is referred to the web version of the article.)

within each hemisphere were highly correlated during each of these 2 periods, with correlation across hemispheres somewhat lower (Fig. 5b, top). The similarity between the two PCorrs can also be readily appreciated (Fig. $5 \mathrm{~b}$, bottom). These patterns of high correlation between channels tended to dominate the dynamics observed, and demonstrated a baseline state where LFP signals from different areas had moderate amplitude activity.

At 43 and $48 \mathrm{~min}$, dark stripes are seen in the PCo matrix, indicating brief periods of anomalous discoordination. These stripes represent a PCorr vector that is very different from the other PCorr vectors in the recording. Fig. $5 \mathrm{c}$ shows the LFP activity and associated PCorr at $43 \mathrm{~min}$ on the left. Correlation with a time at $11 \mathrm{~min}$ was $<0.2$ (red asterisks in Fig. 5a). At the 43 min time, similar to at $48 \mathrm{~min}$, correlation among 3 sites - right and left piriform cortices and right dorsal hippocampus - remained high (Fig. 5c, top left). However, activity between these 3 sites and all other sites was strongly anti-correlated. This demonstrated that these 3 sites had a brief period of activity break-off from other brain locations, suggesting a disruption of normal communication with the rest of the brain. The anomalous PCorr (Fig. 5c, bottom left) is therefore poorly correlated with all other times, such as the time from 11 min (Fig. 5c, right) whose PCorr is similar to PCorrs at other times (compare to those in Fig. 5b).

\section{Discussion}

We developed a technique for measuring the recurrence of neural correlation structure over time, applicable to data recorded from multiple spatial scales of the brain (neuronal ensembles, local field potentials, EEG). The method, $P C o$, evaluates continuous signals, EEG or LFP, but can be readily adapted to look at spike trains by using binning or other methods to create a piecewise continuous signal. The multiple potential uses of $P C o$ suggest that it may become a useful tool to facilitate data-mining of high-dimensional neural datasets across multiple spatial and temporal scales. The technique has the advantage of providing intuitive visualization of complex neural dynamics at full dimensionality.

We used our method to demonstrate the possibility of finding distinct brain states. In the case of hippocampal place-cell ensembles, we could directly show the difference between states because these states correlated directly with the environment in which the animal was placed. However, this analysis was limited to looking grossly at changes in environment between the two different size chambers, due to the confounding factors of the animal's movement in the context of the long window size that was needed due to the low firing rates of these cells. Application of PCo to LFP recordings was able to detect anomalous brain states which 
demonstrated that specific areas of the brain were breaking away from the dynamics of other brain regions.

Recurrence analysis allows comparison of the dynamics of a complex system using embeddings of the time-series (Eckmann et al., 1995). Each embedding is a vector combining information from past states of the system at a given delay, and therefore captures aspects of the system dynamics. By plotting time on the $x, y$ axis and the distance between such embedding vectors as a color, states of the system at different times can be compared visually. This type of technique has been used for assessing physiological data (Webber and Zbilut, 1994), and was able to demonstrate state changes relating to heart-failure (Marwan et al., 2002). Recent work has shown that using embedding vectors is not required for estimating properties of a complex system, such as entropy (Thiel et al., 2004). PCo matrices are a continuation of recurrence analysis, and capture system dynamics without using embedding vectors, but rather using correlation of high-dimensional correlation vectors. These have several advantages over traditional recurrence plots. First, the use of high-dimensional vectors, formed from interactions across multiple areas over sufficient durations, captures more of the system dynamics. Second, the range of values that can be occupied in the PCo matrix are limited to a predefined range (-1-1). This allows for clear visualization on datasets with different properties. Generalizations of the PCo method include using different system interactions in the PCorr vectors. For example, pair-wise mutual information (Rieke et al., 1999) or transfer entropy (Gourevitch and Eggermont, 2007; Marschinski and Kantz, 2002; Schreiber, 2000; Neymotin et al., 2011) between different areas in the vectors, could be used before calculating the PCo matrix. This may provide additional information than is readily accessible with the Pearson correlation, a linear method.

Different brain states, particularly awake vs asleep or vs anesthetized, have different characteristic time scales based on different dominant frequencies, with faster frequencies exhibited in the awake state. In future work, our PCorr/PCo methods could be used with different windows for the different states and could then identify differential coordination between areas across states, recurrence within state, or recurrence of coordination patterns across states.

\section{Acknowledgements}

Supported by grants from the Simons Foundation (294388), and National Institutes of Health: R01EB022903; R01MH084038; R01MH099128; R01MH086638; R42NS064474; U01EB017695.

\section{References}

Allen, E.A., Damaraju, E., Plis, S.M., Erhardt, E.B., Eichele, T., Calhoun, V.D., 2012. Tracking whole-brain connectivity dynamics in the resting state. Cereb. Cortex, bhs352.

Bargmann, C., Newsome, W., Anderson, D., Brown, E., Deisseroth, K., Donoghue, J., MacLeish, P., Marder, E., Normann, R., Sanes, J., et al., 2014. Brain 2025: a scientific vision. Brain Research through Advancing Innovative Neurotechnologies (BRAIN) Working Group Report to the Advisory Committee to the Director. NIH, http://www.nih.gov/science/brain/2025/(US National Institutes of Health, 2014) (accessed 08.10.15)

Bostock, E., Muller, R.U., Kubie, J.L., 1991. Experience-dependent modifications of hippocampal place cell firing. Hippocampus 1 (2), 193-205.

Buzsáki, G., 2010. Neural syntax: cell assemblies, synapsembles, and readers. Neuron 68, 362-385.

Chan, Rosa H.M., Song, Dong, Goonawardena, Anushka V., Bough, Sarah, Sesay, John, Hampson, Robert E., Deadwyler, Sam A., Berger, Theodore W., 2011. Tracking the changes of hippocampal population nonlinear dynamics in rats learning a memory-dependent task. In: 2011 Annual International Conference of the IEEE Engineering in Medicine and Biology Society, pp. 3326-3329.

Chen, J.L., Ros, T., Gruzelier, J.H., 2013. Dynamic changes of ICA-derived EEG functional connectivity in the resting state. Hum. Brain Mapp. 34 (4), 852-868.

Colgin, L.L., Denninger, T., Fyhn, M., Hafting, T., Bonnevie, T., Jensen, O., Moser, M.B., Moser, E.I., 2009. Frequency of gamma oscillations routes flow of information in the hippocampus. Nature 462 (7271), 353-357. de Haan, W., van der Flier, W.M., Wang, H., Van Mieghem, P.F.A., Scheltens, P., Stam, C.J., 2012. Disruption of functional brain networks in Alzheimer's disease: what can we learn from graph spectral analysis of resting-state magnetoencephalography? Brain Connect. 2 (2), 45-55.

Eckmann, J.P., Kamphorst, S.O., Ruelle, D., 1995. Recurrence plots of dynamical systems. Turbul. Strange Attract. Chaos 4 (9), 441.

Fellous, J.M., Tiesinga, PH.E., Thomas, P.J., Sejnowski, T.J., 2004. Discovering spike patterns in neuronal responses. J. Neurosci. 24 (12), 2989-3001.

Fenton, A.A., Kao, H.Y., Neymotin, S.A., Olypher, A., Vayntrub, Y., Lytton, W.W., Ludvig, N., 2008. Unmasking the CA1 ensemble place code by exposures to small and large environments: more place cells and multiple, irregularly arranged, and expanded place fields in the larger space. J. Neurosci. 28 (44), $11250-11262$.

Fenton, A.A., Muller, R.U., 1998. Place cell discharge is extremely variable during individual passes of the rat through the firing field. Proc. Natl. Acad. Sci. U. S. A 95 (6), 3182-3187.

Fukushima, M., Saunders, R.C., Leopold, D.A., Mishkin, M., Averbeck, B.B., 2012. Spontaneous high-gamma band activity reflects functional organization of auditory cortex in the awake macaque. Neuron 74 (5), 899-910.

Gilbert, C.D., Sigman, M., 2007. Brain states: top-down influences in sensory processing. Neuron 54 (5), 677-696.

Gothard, K.M., Skaggs, W.E., McNaughton, B.L., 1996. Dynamics of mismatch correction in the hippocampal ensemble code for space: interaction between path integration and environmental cues. J. Neurosci. 16 (24), 8027-8040.

Gourevitch, B., Eggermont, J.J., 2007. Evaluating information transfer between auditory cortical neurons. J. Neurophysiol. 97 (3), 2533-2543.

Gray, C.M., König, P., Engel, A.K., Singer, W., 1989. Oscillatory responses in cat visual cortex exhibit inter-columnar synchronization which reflects global stimulus properties. Nature 338, 334-337.

Gray, C.M., Maldonado, P.E., Wilson, M., McNaughton, B., 1995. Tetrodes markedly improve the reliability and yield of multiple single-unit isolation from multi-unit recordings in cat striate cortex. J. Neurosci. Methods 63 (1-2), 43-54.

Greicius, M.D., Krasnow, B., Reiss, A.L., Menon, V., 2003. Functional connectivity in the resting brain: a network analysis of the default mode hypothesis. Proc. Natl. Acad. Sci. U. S. A. 100 (1), 253-258.

Greicius, M.D., Supekar, K., Menon, V., Dougherty, R.F., 2009. Resting-state functional connectivity reflects structural connectivity in the default mode network. Cereb. Cortex 19 (1), 72-78.

Haegens, S., Barczak, A., Musacchia, G., Lipton, M.L., Mehta, A.D., Lakatos, P., Schroeder, C.E., 2015. Laminar profile and physiology of the $\alpha$ rhythm in primary visual, auditory, and somatosensory regions of neocortex. J. Neurosci. 35 (42), 14341-14352.

Hagmann, P., Cammoun, L., Gigandet, X., Meuli, R., Honey, C.J., Wedeen, V.J., Sporns, O., 2008. Mapping the structural core of human cerebral cortex. PLoS Biol. 6 (7), e159.

Hebb, D.O., 2002. The Organization of Behavior: A Neuropsychological Theory. Lawrence Erlbaum Associates.

Hunter, M.D., Eickhoff, S.B., Miller, TW.R., Farrow, TF.D., Wilkinson, I.D., Woodruff, PW.R., 2006. Neural activity in speech-sensitive auditory cortex during silence. Proc. Natl. Acad. Sci. U. S. A. 103 (1), 189-194.

Kelemen, E., Fenton, A.A., 2010. Dynamic grouping of hippocampal neural activity during cognitive control of two spatial frames. PLoS Biol. 8 (6), e1000403.

Kelemen, E., Fenton, A.A., 2013. Key features of human episodic recollection in the cross-episode retrieval of rat hippocampus representations of space. PLoS Biol. 11 (7), e1001607.

Kendall, M.G., 1938. A new measure of rank correlation. Biometrika 30 (1-2), $81-93$.

Kentros, C.G., Agnihotri, N.T., Streater, S., Hawkins, R.D., Kandel, E.R., 2004. Increased attention to spatial context increases both place field stability and spatial memory. Neuron 42 (2), 283-295.

Knight, W.R., 1966. A computer method for calculating Kendall's tau with ungrouped data. J. Am. Stat. Assoc. 61 (314), 436-439.

Kudrimoti, H.S., Barnes, C.A., McNaughton, B.L., 1999. Reactivation of hippocampal cell assemblies: effects of behavioral state, experience, and EEG dynamics. J. Neurosci. 19 (10), 4090-4101.

Kühn, S., Gallinat, J., 2013. Resting-state brain activity in schizophrenia and major depression: a quantitative meta-analysis. Schizophr. Bull. 39 (2), 358-365.

Kumar, S., Sedley, W., Barnes, G.R., Teki, S., Friston, K.J., Griffiths, T.D., 2013. A brain basis for musical hallucinations. Cortex.

Lakatos, P., Barczak, A., Neymotin, S.A., McGinnis, T., Ross, D., Javitt, D.C., O'Connell M.N., 2016. Global dynamics of selective attention and its lapses in primary auditory cortex. Nat. Neurosci., http://dx.doi.org/10.1038/nn.4386 [Epub ahead of print].

Lee, H., Dvorak, D., Kao, H.Y., Duffy, ÁM., Scharfman, H.E., Fenton, A.A., 2012. Early cognitive experience prevents adult deficits in a neurodevelopmental schizophrenia model. Neuron 75 (4), 714-724.

Lipska, B.K., Jaskiw, G.E., Weinberger, D.R., 1993. Postpubertal emergence of hyperresponsiveness to stress and to amphetamine after neonatal excitotoxic hippocampal damage: a potential animal model of schizophrenia. Neuropsychopharmacology 9 (1), 67-75.

Louie, K., Wilson, M.A., 2001. Temporally structured replay of awake hippocampal ensemble activity during rapid eye movement sleep. Neuron 29 (1), 145-156.

Lu, H., Zuo, Y., Gu, H., Waltz, J.A., Zhan, W., Scholl, C.A., Rea, W., Yang, Y., Stein, E.A., 2007. Synchronized delta oscillations correlate with the resting-state functional MRI signal. Proc. Natl. Acad. Sci. U. S. A. 104 (46), 18265-18269. 
Lungarella, M., Sporns, O., 2006. Mapping information flow in sensorimotor networks. PLoS Comput. Biol. 2 (10), e144.

Marschinski, R., Kantz, H., 2002. Analysing the information flow between financial time series. Eur. Phys. J. B-Condens. Matter Complex Syst. 30 (2), 275-281.

Marwan, N., Wessel, N., Meyerfeldt, U., Schirdewan, A., Kurths, J., 2002. Recurrence-plot-based measures of complexity and their application to heart-rate-variability data. Phys. Rev. E 66 (2), 26702.

Moncrieff, J., Cohen, D., 2006. Do antidepressants cure or create abnormal brain states? PLoS Med. 3 (7), e240.

Muller, R.U., Kubie, J.L., 1987. The effects of changes in the environment on the spatial firing of hippocampal complex-spike cells. J. Neurosci. 7. 1951-1968.

Neymotin, S.A., Jacobs, K.M., Fenton, A.A., Lytton, W.W., 2011. Synaptic information transfer in computer models of neocortical columns. J. Comput. Neurosci. 30 (1), 69-84.

Neymotin, S.A., Lee, H.Y., Fenton, A.A., Lytton, W.W., 2010. Interictal EEC discoordination in a rat seizure model. J. Clin. Neurophysiol. 27 (6), 438-444

Neymotin, S.A., Lytton, W.W., Olypher, A.O., Fenton, A.A., 2011. Measuring the quality of neuronal identification in ensemble recordings. J. Neurosci. 31 (45), $16398-16409$

O’Keefe, J., 1979. A review of the hippocampal place cells. Prog. Neurobiol. 13 (4), 419-439.

O’Keefe, J., Dostrovsky, J., 1971. The hippocampus as a spatial map. preliminary evidence from unit activity in the freely-moving rat. Brain Res. 34, 171-175.

O’Keefe, J., Recce, M.L., 1993. Phase relationship between hippocampal place units and the EEG theta rhythm. Hippocampus 3 (3), 317-330.

Palva, S., Palva, J.M., 2011. Functional roles of alpha-band phase synchronization in local and large-scale cortical networks. Front. Psychol. 2.

Paxinos, G., Watson, C., 2007. The Rat Brain in Stereotaxic Coordinates, 6th ed. Academic Press, San Diego, CA.

Press, W.H., Teukolsky, S.A., Vetterling, W.T., Flannery, B.P., 2007. Numerical Recipes: The Art of Scientific Computing. Cambridge Univ. Press, Cambridge, MA, USA
Rieke, F., Warland, D., Bialek, W., 1999. Spikes: Exploring the Neural Code. The MIT Press, Cambridge, MA, USA.

Schomburg, E.W., Fernández-Ruiz, A., Mizuseki, K., Berényi, A., Anastassiou, C.A., Koch, C., Buzsáki, G., 2014. Theta phase segregation of input-specific gamma patterns in entorhinal-hippocampal networks. Neuron 84 (2), $470-485$.

Schreiber, T., 2000. Measuring information transfer. Phys. Rev. Lett. 85 (2), 461-464.

Schroeder, C.E., Lakatos, P., 2012. The signs of silence. Neuron 74, 770-772.

Song, D., Chan, R.H., Robinson, B.S., Marmarelis, V.Z., Opris, I., Hampson, R.E., Deadwyler, S.A., Berger, T.W., 2015. Identification of functional synaptic plasticity from spiking activities using nonlinear dynamical modeling. J. Neurosci. Methods 244, 123-135.

Song Dong, Chan, Rosa H.M., Marmarelis, Vasilis Z., Hampson, Robert E., Deadwyler, Sam A., Berger, Theodore W., 2009. Nonlinear modeling of neural population dynamics for hippocampal prostheses. Neural Netw. 22, 1340-1351.

Sporns, O., Zwi, J.D., 2004. The small world of the cerebral cortex. Neuroinformatics 2, 145-162.

Thiel, M., Romano, M.C., Read, P.L., Kurths, J., 2004. Estimation of dynamical invariants without embedding by recurrence plots. Chaos: Interdiscipl. J. Nonlinear Sci. 14, 234

Treisman, A., 1996. The binding problem. Curr. Opin. Neurobiol. 6 (2), 171-178.

Uddin, L.Q., Supekar, K., Lynch, C.J., Cheng, K.M., Odriozola, P., Barth, M.E., Phillips, J., Feinstein, C., Abrams, D.A., Menon, V., 2014. Brain state differentiation and behavioral inflexibility in autism. Cereb. Cortex, bhu161.

von der Malsburg, C., 1981. The correlation theory of brain function (1994 reprint of a report originally published in 1981). In: Domany, E., van Hemmen, J.L., Schulten, K. (Eds.), Models of Neural Networks II. Springer [LAAD].

von der Malsburg, C., 1995. Binding in models of perception and brain function. Neuron 5, 95-104

Webber Jr., C.L., Zbilut, J.P., 1994. Dynamical assessment of physiological systems and states using recurrence plot strategies. J. Appl. Physiol. 76 (2), 965. 